\title{
Towards a consistent eddy-covariance processing: an intercomparison of EddyPro and TK3
}

\author{
G. Fratini ${ }^{1}$ and M. Mauder ${ }^{2}$ \\ ${ }^{1}$ LI-COR Biosciences Inc., Lincoln, Nebraska, USA \\ ${ }^{2}$ Karlsruhe Institute of Technology, Institute of Meteorology and Climate Research - Atmospheric Environmental Research, \\ Garmisch-Partenkirchen, Germany \\ Correspondence to: G. Fratini (gerardo.fratini@licor.com)
}

Received: 17 December 2013 - Published in Atmos. Meas. Tech. Discuss.: 4 March 2014

Revised: 28 May 2014 - Accepted: 24 June 2014 - Published: 29 July 2014

\begin{abstract}
A comparison of two popular eddy-covariance software packages is presented, namely, EddyPro and TK3. Two approximately 1-month long test data sets were processed, representing typical instrumental setups (i.e., CSAT3/LI-7500 above grassland and Solent R3/LI-6262 above a forest). The resulting fluxes and quality flags were compared. Achieving a satisfying agreement and understanding residual discrepancies required several iterations and interventions of different nature, spanning from simple software reconfiguration to actual code manipulations. In this paper, we document our comparison exercise and show that the two software packages can provide utterly satisfying agreement when properly configured. Our main aim, however, is to stress the complexity of performing a rigorous comparison of eddy-covariance software. We show that discriminating actual discrepancies in the results from inconsistencies in the software configuration requires deep knowledge of both software packages and of the eddy-covariance method. In some instances, it may be even beyond the possibility of the investigator who does not have access to and full knowledge of the source code. Being the developers of EddyPro and TK3, we could discuss the comparison at all levels of details and this proved necessary to achieve a full understanding. As a result, we suggest that researchers are more likely to get comparable results when using EddyPro (v5.1.1) and TK3 (v3.11) at least with the setting presented in this paper - than they are when using any other pair of EC software which did not undergo a similar cross-validation.

As a further consequence, we also suggest that, to the aim of assuring consistency and comparability of centralized flux databases, and for a confident use of eddy fluxes
\end{abstract}

in synthesis studies on the regional, continental and global scale, researchers only rely on software that have been extensively validated in documented intercomparisons.

\section{Introduction}

The eddy-covariance (EC) processing sequence to calculate turbulent fluxes from raw, high-frequency data is complex, depending on the chosen instruments, their deployment, the site characteristics and the atmospheric turbulence peculiarities. The software realizing this processing is analogously complex to develop, maintain, document and support. Overviews of popular software packages including detailed lists of their features is available in Foken et al. (2012) and in Aubinet et al. (2012). Such public EC software packages designed and intended for the general public - are repeatedly tested and inter-compared, improved on the basis of users' feedbacks and updated to catch up with new findings and refinements to the EC processing methods. The resulting robustness, quality and reliability are difficult to achieve otherwise.

In contrast, there is a large number of in-house EC software that are typically used by one group only, tailored around a specific EC system, and not intended and designed for the general public. Often, such EC software tend to (1) stagnate (not follow latest developments); (2) age (e.g., being developed for a given platform, they may not be easily ported to new operating systems); (3) not be sufficiently documented; (4) be comprised of difficult-to-read code; (5) not be user-friendly; (6) not be easily extensible to new EC 
systems (e.g., analyzers for new gases). Of course, some in-house software may well not have these characteristics, but considerable effort is required to maintain a high-quality standard and many groups do not have the capacity or resources to do that.

For our purposes, it is convenient to introduce a nomenclature for the operations performed in EC software. In this paper, a processing scheme is the ensemble of all operations performed by the software, from the ingestion of raw data to the calculation of corrected fluxes. A processing step is any major operations in the processing scheme, for example, the tilt correction or the elimination of spikes. For a given processing step, several methods can be available in the literature, and different packages can thus implement a processing step with different methods. Often, a given software supports multiple methods for some of the processing steps, freely selectable by users. It is also to be noted that the same processing scheme can be implemented differently in different packages, because in some cases also the order in which the steps are performed matters. In addition, some software performs iterations of (some) processing steps.

The impact of the entire post-processing typically amounts to 5-20\% for energy fluxes and more than $50 \%$ for $\mathrm{CO}_{2}$ fluxes if open-path analyzers are used (Mauder and Foken, 2006). It is therefore not surprising that fluxes obtained from the same raw data processed with different software packages usually do not agree completely. For energy fluxes, Mauder et al. (2007) found an agreement within 10-15\% in an intercomparison of six different public EC packages from renowned international research institutions, while Mauder et al. (2008) found an agreement within 5-10\% of the resulting $\mathrm{CO}_{2}$ fluxes when comparing seven packages used in CARBOEUROPE-IP. The larger discrepancies in the first study occurred because participants had applied different processing schemes, reflecting different opinions on the best way to process those particular data sets. In contrast, all developers of the second study had followed the same prescribed processing scheme, based on the recommendations of Lee et al. (2004).

A certain agreement was reached by the eddy-covariance community as to which processing steps are necessary under which conditions, thus any EC software can be expected to allow the appropriate processing schemes. However, as mentioned earlier, large uncertainty remains as to which method shall be adopted for each step, and which is the correct order in the processing sequence. Furthermore, plenty of arbitrariness is left to the developers as to how to implement a given method, because typically published papers do not describe methods in sufficient technical detail. Finally, refinements of existing methods and new findings continuously arise, which impose updates to EC software, as documented for example by the recent works on effects of humidity in closedpath measurements (Ibrom et al., 2007b; Fratini et al., 2012; Nordbo and Katul, 2013), angle-of-attack effects (Nakai and Shimoyama, 2012; Kochendorfer et al., 2012; Mauder, 2013) and flux biases due to errors in concentration measurements (Fratini et al., 2014).

Differences in post-processing routines present themselves to the researcher who attempts a software intercomparison as either systematic or random differences in resulting fluxes, which are part of the overall measurement uncertainty and therefore need to be characterized. Richardson et al. (2012) distinguish systematic errors associated with different data processing choices into those that arise from detrending or other kinds of high-pass filtering and those due to the choice of the coordinate rotation method. Moreover, inevitable limitations of instrumentation (e.g., finite time response and averaging volume) require corrections during the post-processing, which may cause additional discrepancies. Instrument-related issues may include spikes, power failure, high-frequency losses and effects of air density fluctuations (Richardson et al., 2012).

Causes for discrepancies during intercomparisons can be conveniently grouped into four classes: (C1) inaccuracies in software configuration that lead to unintended differences in the processing schemes; (C2) differences in the methods available in each software, for any given processing step; (C3) differences in the actual implementation of a given method or differences in the order in which processing steps are implemented; and (C4) implementation errors (bugs).

Class $\mathrm{C} 4$ differs from $\mathrm{C} 2$ and $\mathrm{C} 3$ in that the latter classes are the result of conscious choices of the developer, while bugs (C4) are obviously unintended, and ideally get fixed as soon as they are found. The only class of causes attributable exclusively to the user is $\mathrm{C} 1$. However, while performing an intercomparison, it is crucial to be aware of causes of classes $\mathrm{C} 2$ and $\mathrm{C} 3$, which may require a deep knowledge of the software under consideration and (C3) of its source code.

Assuming no bugs (C4) in the software, in intercomparisons such as the one described in Mauder et al. (2008), cause of class $\mathrm{C} 1$ can be minimized or completely avoided (especially, as we will see, if the comparison is carried out in several iterations). This expectation can be generalized to any intercomparisons carried out by micrometeorologists who are experts on the packages under consideration. In such cases, discrepancies are only due to causes of classes $\mathrm{C} 2$ and C3, which can only be eliminated - if deemed necessary through a modification of either software being tested.

This is however not the general case. Often, for example, when evaluating the possibility of switching to a different software, researchers attempt quick, informal intercomparisons to assess the quality of the new one, only to find that results seldom match to a satisfying degree. As witnessed by the authors, the tendency in this case is to interpret discrepancies as an error in the new software, to which the individual is less accustomed. A deeper investigation, often requiring the intervention of the software developers, in many cases reveals that discrepancies beyond the expectations depicted above are due to causes of class $\mathrm{C} 1$. That is, the software 
configuration was not appropriate to perform a meaningful intercomparison.

Triggered by these considerations, in this paper we present an intercomparison of the two EC public software packages EddyPro and TK3, with the threefold aim of (i) showing that they can give utterly satisfying agreement in calculated fluxes; (ii) identifying the sources of residual discrepancies; (iii) stressing the complexity of performing a fair and rigorous software comparison that highlights genuine discrepancies, which shall eventually be regarded as an ineliminable source of uncertainty. To achieve these aims, we will present the evolution of our comparison, identifying and categorizing the reasons for observed differences, showing how the match improves by elimination of such causes, and discussing residual differences. Note that, while we will occasionally make comments on the suitability of certain implementations, an objective evaluation of alternative methods that we will identify as sources of mismatches is beyond the scope of this work.

EddyPro (www.licor.com/eddypro) and TK3 (Mauder and Foken, 2011) are two of the most popular EC packages that are freely available, with about 3300 downloads in over 150 countries and more than 870 downloads in more than 53 countries to date.

EddyPro is a free of charge, open-source software released by LI-COR Biosciences Inc. (Lincoln, NE, USA) under the GPL license. It was firstly released in April 2011 as EddyPro Express 2.0. Its code base builds entirely on $\mathrm{ECO}_{2} \mathrm{~S}$ (the Eddy Covariance Community Software), an open-source software project started in 2007 at the University of Tuscia (Viterbo, Italy) and partially funded by the IMECC (http://imecc.ipsl.jussieu.fr/) and ICOS (www.icos-infrastructure.eu) European projects. Before release, $\mathrm{ECO}_{2} \mathrm{~S}$ was officially tested in a software intercomparison and results are documented in an IMECC project report. At the time of writing this paper, EddyPro version 5.1.1 included various options for each processing step required in the eddy-covariance chain.

The history of TK3 can be traced back over more than twenty years. It started with the Turbulenzknecht program which was first used to automatically compute turbulent fluxes in 1989. Its major assets were the elaborate quality assessment routines, which were unique at the time (Foken and Wichura, 1996). After more than ten years of successful application in many micrometeorological field campaigns the software was redeveloped from scratch in order to utilize the rapid advancements in computer technology and to allow for automatic processing of much longer data sets for up to one year. The resulting TK2 software included all state of the art flux corrections (Lee et al., 2004) and was extensively compared with other publically available EC-software (Mauder et al., 2008). Its updated version TK3 is in continuation of this lineage. TK3 is technically not open-source software; however, selected parts of the code can be made available for inspection upon request.
We started our comparison using EddyPro v.5.0 and TK3 v3.11. As we will see, the intercomparison triggered some modifications to EddyPro which are already available in the current version 5.1.1, while the implementation of the despiking method of Mauder et al. (2013) in EddyPro is planned for a forthcoming release (see Sect. 3.1).

\section{Comparison strategy}

Two test data sets were selected with the intention to be representative of long-term flux observation setups (see Table 1). They both cover a period longer than 1 month in order to represent different weather conditions during the growing season. The closed-path data set originates from the Hainich EC station above a beech forest, which was part of the CARBOEUROPE-IP network (Knohl et al., 2003). This system consists of a Solent R3 sonic anemometer (Gill Instruments Ltd., UK) and a LI-6262 closed-path gas analyzer (LI-COR Biosciences) at a measurement height of $45 \mathrm{~m}$. The open-path data set is from an EC system above a grassland located near Graswang, Germany (Mauder et al., 2013), which is part of the Terrestrial Environmental Observatories network TERENO (Zacharias et al., 2011). The measurement height was $3.1 \mathrm{~m}$ and the instrumentation consisted of a CSAT3 sonic anemometer (Campbell Scientific Inc., Logan, UT, USA) and a LI-7500 open-path gas analyzer (LI-COR Biosciences Inc.).

In our comparison, we considered results obtained for friction velocity $\left(u_{*}, \mathrm{~ms} \mathrm{~s}^{-1}\right), \mathrm{CO}_{2}$ fluxes $\left(F_{\mathrm{c}}, \mu \mathrm{mol} \mathrm{m}{ }^{-2} \mathrm{~s}^{-1}\right)$, latent heat fluxes $\left(\mathrm{LE}, \mathrm{W} \mathrm{m}^{-2}\right)$, sensible heat fluxes $(H$, $\mathrm{W} \mathrm{m}^{-2}$ ) and all corresponding quality flags according to the CARBOEUROPE-IP 0/1/2 scheme (Foken et al., 2004). We intentionally started the comparison with a generic definition of the processing scheme, of the kind that an average user would make. We stress again that, having full knowledge and control of the software code, we could in principle agree on the finest details at the onset, and have the software provide the exact same results at the first trial (provided the codes are free of bugs), but this would be of little help as it would not replicate any realistic situation. Instead, we strived to simulate the typical starting point of an investigator who attempts an intercomparison, thus assuming proper knowledge of the EC method and of configuration of the two software packages, but not sufficient control over the source code. In fact, having access to the source code of software packages comprised of tens of thousands of code lines does not automatically grant the ability to understand it or meaningfully and safely modify it. We note here that this consideration applies to any EC software, both "public" and "in-house" as per the definitions provided in the introduction.

After a first round, we analyzed the results and refined the comparison in two more rounds, by sorting out all potential causes of class $\mathrm{C} 1$ (i.e., the eliminable differences due to the configuration of the processing scheme) as well as causes of 
Table 1. Overview of the two test data sets: measured variables were wind components $u, v$ and $w$, sonic temperature $T_{\mathrm{S}}, \mathrm{CO}_{2}$ and $\mathrm{H}_{2} \mathrm{O}$ concentration (either number densities or mole fractions) and air pressure $p$.

\begin{tabular}{lll}
\hline Data set & closed-path & open-path \\
Duration & 49 days & 38 days \\
Variables & $u, v, w, T_{\mathrm{s}}, \mathrm{CO}_{2}, \mathrm{H}_{2} \mathrm{O}$ & $u, v, w, T_{\mathrm{S}}, \mathrm{CO}_{2}, \mathrm{H}_{2} \mathrm{O}, p$ \\
Instruments & Solent R3/LI-6262 & CSAT3/LI-7500 \\
Ecosystem & Forest & Grassland \\
Measurement height & $19 \mathrm{~m}$ & $3.1 \mathrm{~m}$ \\
\hline Tilt correction & Planar fit & Double rotation \\
\hline
\end{tabular}

class $\mathrm{C} 2$ and $\mathrm{C} 3$ (i.e., differences intrinsic to the software). In a couple of cases this exercise led to a revision/extension of either software, while some differences, assessed as being of class $\mathrm{C} 2$ (different methods for the same processing step), did remain and fully account for the residual differences.

After improving the match with the closed-path data set, we may have expected to need less than three rounds for the open-path one. However, open- and closed-path data exercise rather different parts of the code and for this reason we decided to do three rounds in both cases.

We decided to apply two different tilt corrections for the two data sets: double rotation for the open-path system and planar fit for the closed-path system (Wilczak et al., 2001) in order to test the agreement between the two packages with both methods. In accordance with the recommendations of Aubinet et al. (2012), we agreed on the processing scheme described in Table 2.

\section{Results and discussion}

The quality of the match between EddyPro and TK3 was quantified by deriving linear regressions (slope, intercept and $r^{2}$ ) of the scatter plots of individual fluxes. Note that the choice of $x$ and $y$ axis for EddyPro and TK3 in the scatter plots was arbitrary and that - because of the independence of the two data sets - we opted for the symmetric RMA (reduced major axis) linear regression model. Furthermore, we considered the percentage of flux results for which the quality flags matched. Rather than presenting only the result of the last round, in the following we shortly describe results obtained during the three rounds, to highlight the reasons for discrepancies and how we could improve the match in subsequent rounds. What we want to stress here is that some improvements were achieved by better tuning the configurations in order to perform the same operations in both EddyPro and TK3, while other improvements could only be achieved by intervening on the source code.

\subsection{Closed-path data set}

In the first round, results from the closed-path data set showed a general close agreement, however accompanied by a significant number of scattering fluxes (Fig. 1). In addition, $F_{\mathrm{c}}$ showed a relatively large systematic bias $(8 \%)$ and the match of calculated quality flags was very poor: for $u_{*}$, LE and $F_{\mathrm{c}}$, only about $60 \%$ of the obtained fluxes received the same quality flag from both packages. Only for $H$, the agreement was almost $90 \%$ already.

Investigation revealed that one major difference of class $\mathrm{C} 2$ was hidden in the despiking processing step. In fact, TK3 implements the robust statistical method of Mauder et al. (2013) based on median absolute deviation (MAD), while the Gaussian statistical method of Vickers and Mahrt (1997) was used in EddyPro. This difference explained most of the observed scatter. Visual inspection and analysis of flux variances showed that for those scattering points EddyPro results were the implausible ones, giving variances $>10$ times larger than those of TK3, which instead fell into plausible ranges. Clearly, for those cases the despiking algorithm of Vickers and Mahrt (1997) - at least with the default settings used - was ineffective to remove large spikes in the raw data, which compromised the flux values. Since the newer despiking method of Mauder et al. (2013) proved to be more effective in our case, the same algorithm was also implemented in EddyPro. Hence, the scatter was largely eliminated in the second round for all observed fluxes. Changes in the source code of EddyPro were required to make this possible, and the new implementation will be available to EddyPro users as an alternative despiking method in a forthcoming release. In the second round, the agreement between quality flags also slightly improved because of the improved comparability of the two packages after this modification. It is to be noted that the despiking method of Vickers and Mahrt (1997) is highly customizable. Therefore, we could have followed a different strategy and try and fine-tune that method in EddyPro until results matched satisfyingly. However, because of the soundness and simplicity of the MAD method, it was deemed appropriate to implement it in EddyPro and propose it as an option to its users.

On the basis of past experience, in the third round we speculated that the systematic bias in $F_{\mathrm{c}}$ could be related to the WPL term and actually, by a mere matter of communication, we found that TK3 and EddyPro were set to treat gas concentration data differently: concentration data were intended 
Table 2. Processing scheme for the software intercomparison

\begin{tabular}{ll}
\hline Raw data preparation & - Elimination of spikes \\
& - Elimination of outranged values, based on physical thresholds \\
\hline Raw data processing & -30 min block averaging \\
& - Cross-wind correction as applicable \\
& - Coordinate transformations \\
& - Time lag compensation (covariance maximization in a predefined window) \\
\hline Flux correction & - Correction for density fluctuations as applicable \\
& - Correction for high-frequency spectral losses \\
& - Humidity correction of sensible heat flux from sonic temperature measurements \\
\hline QA/QC & - According to Foken and Wichura (1996) and Foken et al. (2004) \\
\hline
\end{tabular}

as dry mole fractions (also called mixing ratios, moles of gas per mole of dry air) in TK3 and as mole fractions (moles of gas per mole of air) in EddyPro. Far from being a special occurrence in our comparison, it is often the case that concentration data are available without a clear indication of whether measurements are expressed as mole fractions or as dry mole fractions, as both units are normally reported as $\mu \mathrm{mol} \mathrm{mol}^{-1}$ (or ppm) for $\mathrm{CO}_{2}$ and as $\mathrm{mmol} \mathrm{mol}^{-1}$ for $\mathrm{H}_{2} \mathrm{O}$. The difference between the two is the dilution effect of $\mathrm{H}_{2} \mathrm{O}$ on $\mathrm{CO}_{2}$ measurements (Webb et al., 1980; Ibrom et al., 2007a). Thus, in this case we confronted ourselves with a difference of class $\mathrm{C}$, a rather trivial but utterly common difference in the settings. Clearly agreeing on the nature of the measurements (which happened to be dry mole fractions) was sufficient to improve the slope of the $F_{\mathrm{c}}$ regression by $6 \%$, from 0.92 to 0.97 . Interestingly, we noted that this adjustment had a negative effect on LE comparison, which exhibited a slope of 0.99 in the second round, and of 0.97 in the last one. Evidently, the seemingly perfect initial match was the result of systematic differences contributing in opposite directions and largely offsetting each other.

The differences observed in the calculated quality flags required deeper investigation, and highlighted several differences in the implementation: (i) the steady-state test was evaluated at different stages in the processing scheme; (ii) the quantities involved in the flag definition were slightly different; and (iii) the definition of the flag for the integral turbulence characteristics (ITC) was following different references: Foken et al. (2004) in TK3 and Göckede et al. (2004) in EddyPro. The agreement of the quality flags was greatly improved by reconsideration of these aspects in EddyPro. The group of TK3 developers has a long tradition in the definition of these quality flags, thus it was deemed appropriate to adapt EddyPro towards TK3, rather than the opposite. Nonetheless, residual discrepancies remained (up to $20 \%$ for the quality flags of $F_{\mathrm{c}}$ ) because difference (iii) was not addressed. It is to be noted that, being both based on peerreviewed published materials, there is no objective way to define a "better" implementation, so this difference (of class C2) shall be regarded as a source of ineliminable uncertainty.
Similarly, minor differences in the actual implementation of the quality flag assessment (for example, in EddyPro the stationarity test is evaluated after coordinate rotation for tilt correction, while in TK3 it is evaluated before that processing step: a difference of class $\mathrm{C} 3$ ) contribute to this uncertainty.

The remaining differences in $F_{\mathrm{c}}$ and LE (about $3 \%$ ) are entirely explained by different spectral correction methods. TK3 implements the method of Moore (1986), based on analytical transfer functions. Here, tube-dampening effects were taken into account by a first-order filter transfer function with $2 \mathrm{~Hz}$ cutoff frequency and the sensor separation in lateral direction was corrected according to Moore's transfer function while the longitudinal separation had already been eliminated by the time lag compensation. EddyPro supports several spectral correction schemes, including a few accounting for relative humidity $(\mathrm{RH})$ dependent effects on water vapor fluxes (Ibrom et al., 2007b; Fratini et al., 2012). For the current comparison, the method described in Horst (1997) was selected because, among the ones not accounting for $\mathrm{RH}$ effects, it is the only one for which a cutoff frequency of $2 \mathrm{~Hz}$ could be prescribed a priori. Effects of lateral separation were accounted for following the method of Horst and Lenschow (2009). For closed-path data, the spectral correction is the last step in the chain (possibly before an iteration of the corrections, which was however not performed in our comparison) thus in this case it was easy to verify that the different correction factors provided by different methods fully explained the residual difference in $F_{\mathrm{c}}$ and LE which, again, shall be regarded as an intrinsic uncertainty.

\subsection{Open-path data set}

The comparison with the open-path data set benefitted from the experience gained during the previous comparison and from modifications in the settings and revised implementations (namely, despiking and quality flags, see Sect. 3.1). As a consequence, during the three rounds with this second data set we did not observe improvements in the match of quality flags. 

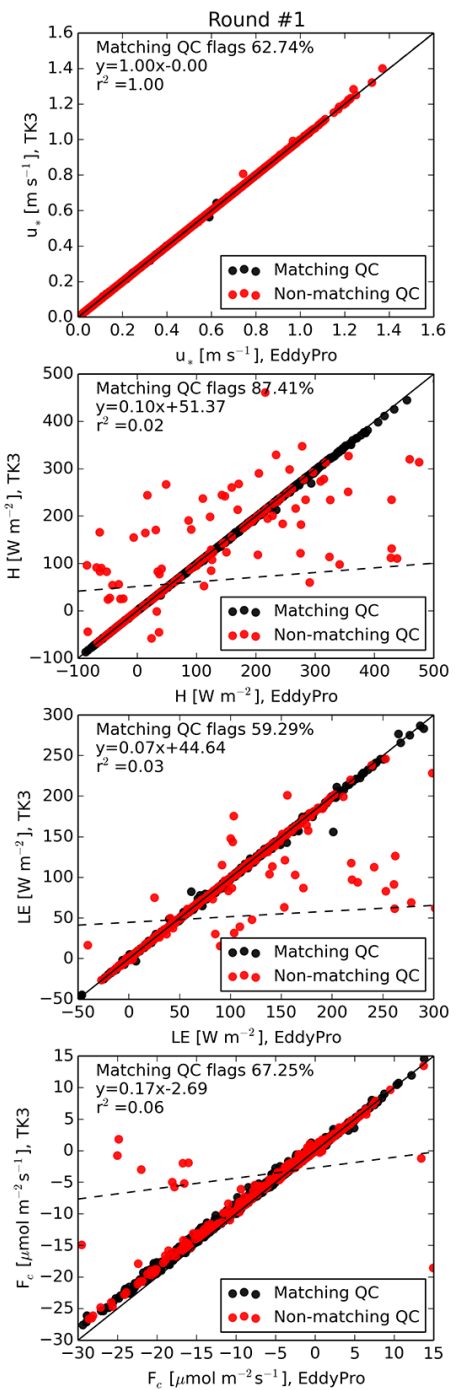
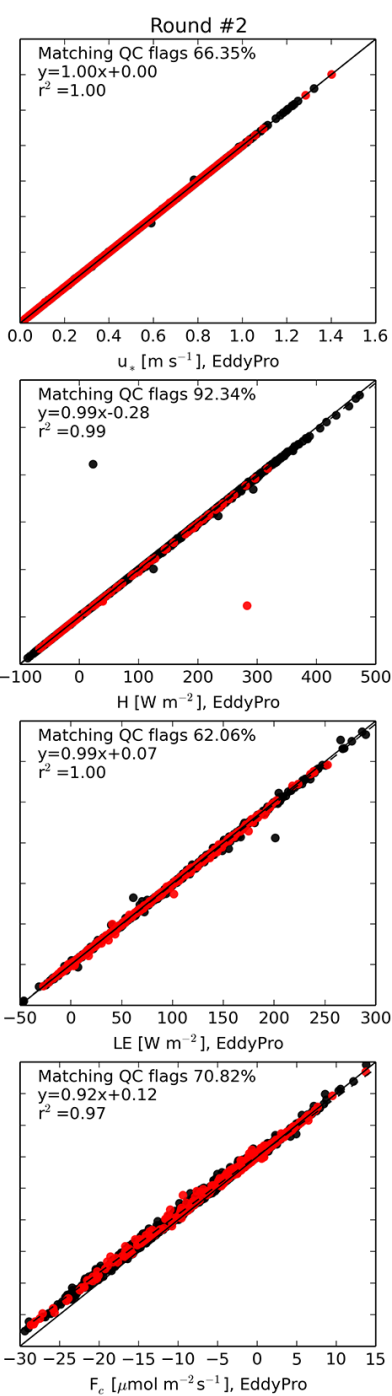
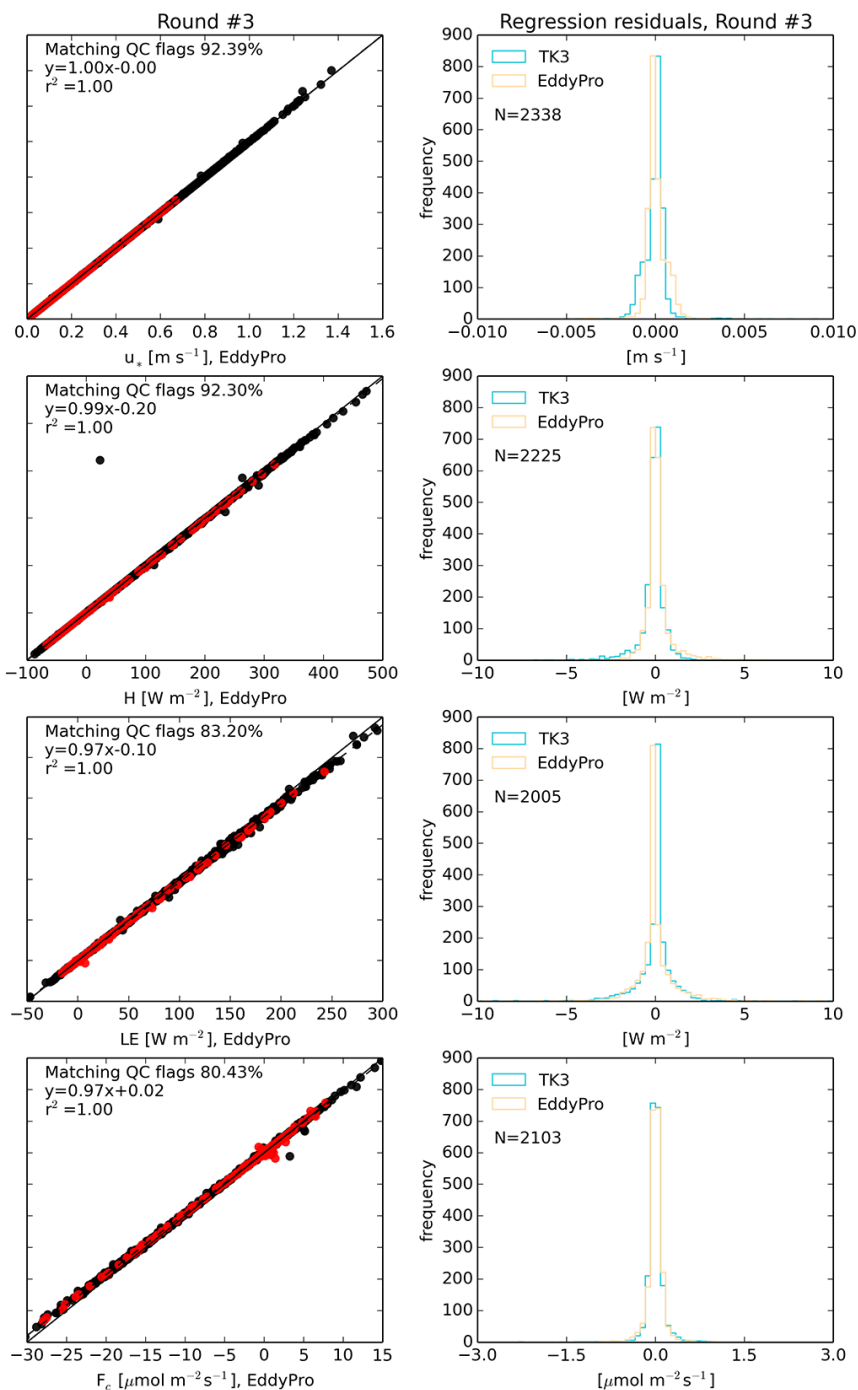

Figure 1. Scatter plots including RMA regression parameters for $u_{*}, H, \mathrm{LE}$ and $F_{\mathrm{c}}$, for the closed-path data set calculated with EddyPro and TK3. The results of the three comparison rounds with refined software configurations are displayed from left to right. Very poor regression parameters in the first round (leftmost plots) are driven by wildly scattering data points, lying outside the chart areas. The rightmost column shows the residuals of the linear regression for the third round.

During the first round, we observed a significant dispersion, particularly for $F_{\mathrm{c}}$, and a systematic underestimation of $F_{\mathrm{c}}$ and LE in TK3 as compared to EddyPro (Fig. 2). The following discussion highlighted that EddyPro was using barometric pressure (as estimated by site altitude) while TK3 was using pressure data available in the raw data files. That is, we incurred another discrepancy of class $\mathrm{C} 1$, and one worth discussing. The pressure data in the raw files was not accompanied by metadata detailing its meaning, units and relevance to the eddy-covariance data. In this situation, the natural way of proceeding in EddyPro is to ignore this data and use barometric pressure instead. More in general, it is good practice to ignore data that are not fully documented. For example, in a closed-path system a pressure data may refer to, at least, the ambient air or the instrument's cell: interpreting this data in the wrong way would lead to significant systematic biases in fluxes. Seen from the opposite perspective, we suggest always combining raw data with the metadata necessary to correctly interpret it and use it during flux computation.

Once the software were set to use the pressure data from the raw files in the second round, most of the scatter was eliminated and we were left with systematic differences in $F_{\mathrm{c}}$ and LE of about 5-6\%. The agreement in quality flags is better than that at the first round of the closed-path comparison with almost $70 \%$ matching flags for all fluxes and even $92 \%$ matching flags for $H$.

Similar to the closed-path systems comparison, it was easy to guess that the difference was due to different spectral correction procedure. TK3 used again the correction from Moore (1986), while this time the analytic method of 

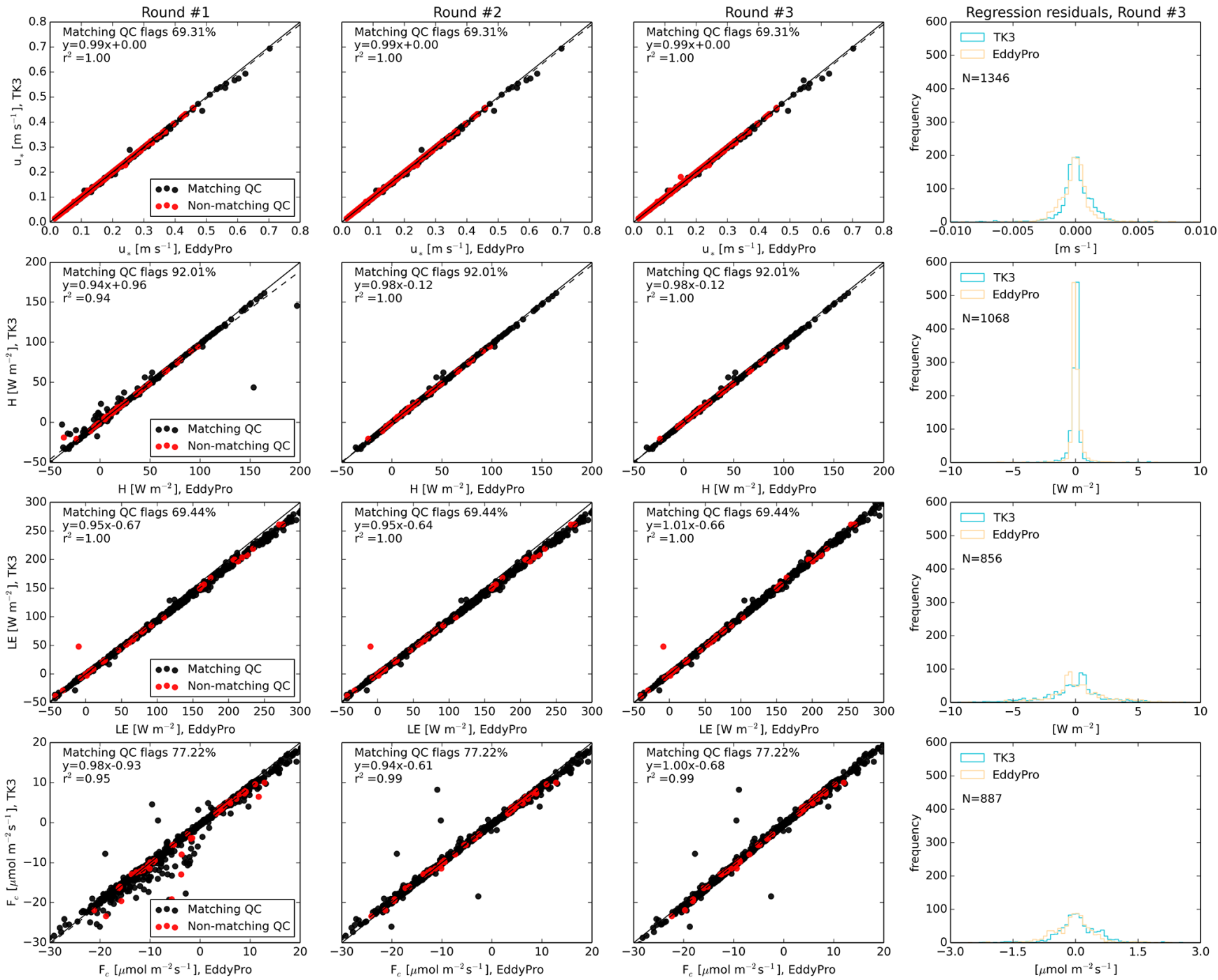

Figure 2. Same as Fig. 1, but for the open-path data set.

Moncrieff et al. (1997) was used in EddyPro. Different from the closed-path case, however, open-path data presents an additional complication when trying to entangle the effects of different spectral corrections from other potential sources of discrepancies. In fact, in this case the WPL terms - which are additive in nature - must be included after all fluxes have been corrected for spectral attenuations, and spectral corrections are thus no longer the last step in the processing scheme. To verify whether the difference in the spectral corrections solely accounted for the whole observed difference, in the third round we artificially (and only temporarily) modified EddyPro to match on average (i.e., across the whole data set) the spectral correction factors calculated by TK3. After this operation, any residual systematic difference would have to be attached to the treatment of the WPL terms. Obviously, this manipulation is only possible if one has full control over (i.e., not only access to, but also appropriate knowledge of) the software source code, while it would be relatively difficult and error prone trying to do the same by proceeding backward from final fluxes.

Results show a largely satisfying agreement, with systematic differences virtually eliminated and limited scatter due to residual differences in the spectral corrections. We note again, however, that such agreement cannot be achieved by the normal user of the software who cannot (or is not able to) modify the source code to force - as we did - the spectral correction factors to match, before the WPL terms are calculated. We also note again that the open-path data set obtained over grassland was processed using double rotation while the closed-path data set obtained above a forest was processed using planar-fit tilt correction. While the doublerotation method has a relatively simple and unambiguous definition, the planar-fit method is significantly more complex and leaves the developer with some degree of freedom 
as to how to implement it. The good matches achieved with both methods across the two data sets after the three rounds show that the implementations of TK3 and EddyPro are consistent, providing a sound cross-validation.

\section{Conclusions}

We have shown that, when properly configured, the two software packages EddyPro and TK3 provide satisfying, yet not perfect, agreement in calculated fluxes and related quality flags. Initial comparisons highlighted discrepancies that could be eliminated by simply improving communication, exchanging more details on data significance and on the processing scheme. This suggests the importance of a very detailed consensus on EC post-processing to achieve the best possible comparability between fluxes processed by different users, even when using the same software, and of a very careful setup when an individual attempts the comparison between two software packages.

Achieving further improvement required interventions on the source code, in particular with the implementation of the spike detection algorithm of Mauder et al. (2013) in EddyPro, which is soon to become a standard option also in this software. The spectral correction procedures are quite different between EddyPro (Horst, 1997; Moncrieff et al., 1997) and TK3 (Moore, 1986). This is the processing step that caused the largest differences in flux results, differences that could not be eliminated using the current versions of the software. This finding suggests that further effort in the eddy-covariance methodology shall aim at reducing systematic discrepancies obtained with different spectral correction approaches and methods.

Residual differences in quality flags were mostly due to different algorithms used for the well-developed turbulence test (Foken et al., 2004).

From our exercise, we conclude that discriminating among actual implementation errors, intentional differences and inaccuracies in the software configuration may only be possible to the investigator who has detailed knowledge of the source code and the ability to apply appropriate changes. The presented comparison did not highlight any obvious bug (C4). All differences observed in the third round are explained in terms of different implementations of the same methods, or to the adoption of different methods. As a result of this effort and considering the results obtained in previous intercomparisons (Mauder et al., 2007, 2008), we suggest that researchers are now more likely to get comparable results when using EddyPro (v5.1.1 and above) and TK3 (v3.11), than they are when using any other pair of EC software which did not undergo a similar cross-validation.

Generalizing our findings, we also conclude that an exhaustive documentation of how fluxes are calculated from raw data should, whenever possible, include the details of the adopted processing scheme and possibly the name and version of software used. Finally, we want to warn against ad hoc software intercomparisons as a means to validate (or invalidate) EC software, unless they are carried out by experts of the software under considerations and with the required level of detail, as demonstrated here. For the same reasons, when flux accuracy is of importance, we warn against the use of in-house software, if this does not undergo systematic quality assurance procedures. In order to assure consistency and comparability of centralized flux databases, we rather suggest researchers to rely on public software packages, notably those that are continuously QA/QC screened, and extensively validated in documented intercomparisons (e.g., Mauder et al., 2008, and this paper).

Acknowledgements. We wish to thank Mathias Herbst for providing the raw data set from the Hainich station. M. Mauder's contribution was partly funded by the Helmholtz-Association through the President's Initiative and Networking Fund. TERENO is funded by the Helmholtz Association and the German Federal Ministry of Education and Research.

Edited by: S. Malinowski

\section{References}

Aubinet, M., Vesala, T., and Papale, D. (Eds): Eddy Covariance: A Practical Guide to Measurement and Data Analysis, Springer, Berlin, 460 pp., 2012.

Foken, T. and Wichura, B.: Tools for quality assessment of surfacebased flux measurements, Agr. Forest Meteorol., 78, 83-105, 1996.

Foken, T., Göckede, M., Mauder, M., Mahrt, L., Amiro, B. D., and Munger, J. W.: Post-field data quality control, in: Handbook of Micrometeorology. A Guide for Surface Flux Measurements, edited by: Lee, X., Massman, W. J., and Law, B. E., Kluwer, Dordrecht, 181-208, 2004.

Foken, T., Leuning, R., Oncley, S. P., Mauder, M., and Aubinet, M.: Corrections and data quality, in: Eddy Covariance: A Practical Guide to Measurement and Data Analysis, edited by: Aubinet, M., Vesala, T., and Papale, D., Springer, Dordrecht, 85-132, 2012.

Fratini, G., Ibrom, A., Arriga, N., Burba, G., and Papale, D.: Relative humidity effects on water vapour fluxes measured with closed-path eddy-covariance systems with short sampling lines, Agr. Forest Meteorol., 165, 53-63, 2012.

Fratini, G., McDermitt, D. K., and Papale, D.: Eddy-covariance flux errors due to biases in gas concentration measurements: origins, quantification and correction, Biogeosciences, 11, 1037-1051, doi:10.5194/bg-11-1037-2014, 2014.

Göckede, M., Rebmann, C., and Foken, T.: A combination of quality assessment tools for eddy covariance measurements with footprint modelling for the characterisation of complex sites, Agr. Forest Meteorol., 127, 175-188, 2004.

Horst, T. W.: A simple formula for attenuation of eddy fluxes measured with first-order-response scalar sensors, Bound.-Lay. Meteorol., 82, 219-233, 1997. 
Horst, T. W. and Lenschow, D. H.: Attenuation of scalar fluxes measured with spatially-displaced sensors, Bound.-Lay. Meteorol., 130, 275-300, doi:10.1007/s10546-008-9348-0, 2009.

Ibrom, A., Dellwik, E., Larsen, S. E., and Pilegaard, K.: On the use of the Webb-Pearman-Leuning theory for closed-path eddy correlation measurements, Tellus B, 59, 937-946, 2007a.

Ibrom, A., Dellwik, E., Flyvbjerg, H., Jensen, N. O., and Pilegaard, K.: Strong low-pass filtering effects on water vapour flux measurements with closed-path eddy correlation systems, Agr. Forest Meteorol., 147, 140-156, 2007b.

Knohl, A., Schulze, E. D., Kolle, O., and Buchmann, N.: Large carbon uptake by an unmanaged 250-year-old deciduous forest in Central Germany, Agr. Forest Meteorol., 118, 151-167, 2003.

Kochendorfer, J., Meyers, T. P., Frank, J., Massman, W. J., and Heuer, M. W.: How well can we measure the vertical wind speed? Implications for fluxes of energy and mass, Bound.-Lay. Meteorol., 145, 383-398, 2012.

Lee, X., Massman, W., and Law, B. E. (Eds): Handbook of Micrometeorology, A Guide for Surface Flux Measurement and Analysis, Kluwer Academic Press, Dordrecht, 250 pp., 2004.

Mauder, M.: A comment on "How well can we measure the vertical wind speed? Implications for fluxes of energy and mass" by Kochendorfer et al., Bound.-Lay. Meteorol., 147, 329-335, 2013.

Mauder, M. and Foken, T.: Impact of post-field data processing on eddy covariance flux estimates and energy balance closure, Meteorol. Z, 15, 597-609, 2006.

Mauder, M. and Foken, T.: Documentation and Instruction Manual of the Eddy-Covariance Software Package TK3. Universität Bayreuth, Abteilung Mikrometeorologie 46, ISSN 1614-8924, 60 pp., 2011.

Mauder, M., Oncley, S. P., Vogt, R., Weidinger, T., Ribeiro, L., Bernhofer, C., Foken, T., Kohsiek, W., de Bruin, H. A. R., and Liu, H.: The Energy Balance Experiment EBEX-2000. Part II: Intercomparison of eddy-covariance sensors and post-field data processing methods, Bound.-Lay. Meteorol., 123, 29-54, 2007.

Mauder, M., Foken, T., Clement, R., Elbers, J. A., Eugster, W., Grünwald, T., Heusinkveld, B., and Kolle, O.: Quality control of CarboEurope flux data - Part 2: Inter-comparison of eddy-covariance software, Biogeosciences, 5, 451-462, doi:10.5194/bg-5-451-2008, 2008
Mauder, M., Cuntz, M., Drüe, C., Graf, A., Rebmann, C., Schmid, H. P., Schmidt, M., and Steinbrecher, R.: A strategy for quality and uncertainty assessment of long-term eddy-covariance measurements, Agr. Forest Meteorol., 169, 122-135, 2013.

Moncrieff, J. B., Massheder, J. M., DeBruin, H., Elbers, J., Friborg, T., Heusinkveld, B., Kabat, P., Scott, S., Søgaard, H., and Verhoef, A.: A system to measure surface fluxes of momentum, sensible heat, water vapor and carbon dioxide, J. Hydrol., 188-189, 589-611, 1997.

Moore, C. J.: Frequency response corrections for eddy correlation systems, Bound.-Lay. Meteorol., 37, 17-35, 1986.

Nakai, T. and Shimoyama, K.: Ultrasonic anemometer angle of attack errors under turbulent conditions, Agr. Forest Meteorol., 162-163, 14-26, 2012.

Nordbo, A. and Katul, G.: A Wavelet-Based Correction Method for Eddy-Covariance High-Frequency Losses in Scalar Concentration Measurements, Bound.-Lay. Meteorol., 146, 81-102, 2013.

Richardson, A. D., Aubinet, M., Barr, A. G., Hollinger, D. Y., Ibrom, A., Lasslop, G., and Reichstein, M.: Uncertainty quantification, in: Eddy Covariance: A Practical Guide to Measurement and Data Analysis, edited by: Aubinet, M., Vesala, T., and Papale, D., Springer, Dordrecht, 173-210, 2012.

Vickers, D. and Mahrt, L.: Quality control and flux sampling problems for tower and aircraft data, J. Atmos. Ocean. Technol., 14, 512-526, 1997.

Webb, E. K., Pearman, G. I., and Leuning, R.: Correction of the flux measurements for density effects due to heat and water vapour transfer, Q. J. Roy. Meteorol. Soc., 106, 85-100, 1980.

Wilczak, J. M., Oncley, S. P., and Stage, S. A.: Sonic anemometer tilt correction algorithms, Bound.-Lay. Meteorol., 99, 127-150, 2001.

Zacharias, S., Bogena, H., Samaniego, L., Mauder, M., Fuß, R., Pütz, Th., Frenzel, M., Schwank, M., Baessler, C., ButterbachBahl, K., Bens, O., Borg, E., Brauer, A., Dietrich, P., Hajsek, I., Helle, G., Kiese, R., Kunstmann, H., Klotz, St., Munch, J. C., Papen, H., Priesack, E., Schmid, H. P., Steinbrecher, R., Rosenbaum, U., Teutsch, G., and Vereecken, H.: A network of terrestrial environmental observatories in Germany, Vadoze Zone J., 10, 955-973, 2011. 